\title{
The knowledge, attitude and practice of hospital pharmacists on the safety issues of antitumor agents for the patients discharged in China
}

\author{
Xiuli Xu\#, Yuhui Yang", Qianqian Fan, Zhu Zhu \\ Department of Pharmacy, Peking Union Medical College Hospital, Chinese Academy of Medical Sciences, Beijing, China \\ Contributions: (I) Conception and design: Z Zhu, Y Yang; (II) Administrative support: Q Fan, Z Zhu; (III) Provision of study materials or patients: Y \\ Yang; (IV) Collection and assembly of data: Y Yang, X Xu; (V) Data analysis and interpretation: X Xu, Y Yang, Q Fan; (VI) Manuscript writing: All \\ authors; (VII) Final approval of manuscript: All authors. \\ \#These authors contributed equally to this work. \\ Correspondence to: Zhu Zhu. Department of Pharmacy, Peking Union Medical College Hospital, Chinese Academy of Medical Sciences, No.1 \\ Shuaifuyuan, Wangfujing, Dongcheng District, Beijing, China. Email: zhuzhu@pumch.cn.
}

\begin{abstract}
Background: Patient safety is a serious global public health concern, especially for those patients undergoing chemotherapy. The purpose of this pilot study was to assess the knowledge, attitude, and practice among hospital pharmacists in China towards the safety issues of antitumor agents for the patients discharged.

Methods: The pilot study was processed using an online questionnaire targeting hospital pharmacists in China. Ten, five and four questions were used to investigate pharmacists' knowledge, attitude and practice (KAP) respectively.

Results: A total of 360 questionnaires by pharmacists out of $412(87.4 \%)$ were qualified and enrolled. It was found that $58.7 \%$ were familiar with safety-related knowledge; $32.2 \%$ believed that safety risks exist and $85.3 \%$ were willing to provide medication education to patients with cancer who take medication at home after discharge; $91.4 \%$ have sometimes taken the initiative to promote safety-related knowledge to their patients.

Conclusions: The research firstly provided some insights into the hospital pharmacists' KAP regarding the safety issues of antitumor agents for patients with cancer discharged in China. Attention should be paid to the knowledge of pharmacists, the awareness of the safety risks of patients with cancer who take medication at home after discharge, and the accessibility to consult with pharmacists after discharge.
\end{abstract}

Keywords: Hospital discharge; patients with cancer; antitumor agents; patient safety; pharmacists' knowledgeattitude-practice

Submitted Jun 28, 2020. Accepted for publication Nov 16, 2020.

doi: $10.21037 /$ tcr-20-2418

View this article at: http://dx.doi.org/10.21037/tcr-20-2418

\section{Introduction}

Malignant tumors have become a common disease endangering the health of human being. According to GLOBOCAN2018, there are approximately 18.08 million new cases of malignant tumors and 9.56 million deaths worldwide. China accounts for approximately $23.7 \%$ and $30 \%$, respectively $(1,2)$. A majority of cancer treatment strategies are based on the removal of tumor mass mainly by surgery. chemotherapy and radiotherapy have also made a major contribution in inhibiting rapid growth of malignant cells. Furthermore, these approaches are often combined to enhance therapeutic indices (3). Currently, cancer treatment is a highly dynamic field and significant advances are being made in the development of novel cancer treatment 
strategies. In contrast to conventional cancer therapeutics, novel approaches such as ligand or receptor-based targeting, triggered release, intracellular drug targeting, gene delivery, cancer stem cell therapy, cancer immunotherapy, magnetic drug targeting and ultrasound-mediated drug delivery, have added new modalities for cancer treatment (4-6). The ultimate goal of cancer therapy is to optimize the efficacy, reduce the systemic toxicity and improve the quality of life. Fortunately, survival rates of many types of cancers are improving owing to the accessible early detection, quality treatment and survivorship care in recent years (7). Survivors with tumors have higher requirements for quality of life, and the treatment and rehabilitation of patients have gradually extended from the hospital to the community and family (8). Antitumor agents for the patients with cancer discharged, which are known as high-alert drugs or cytotoxic drugs, are often administered to immunocompromised patients, and as most of these drugs are myelosuppressive this may place patients at a high risk of developing severe infections (9). In addition, discharged patients with cancer are in a treatment gap due to lack of supervision from medical staff $(10,11)$, and they barely know the principles of safe handling, and dealing with spills, waste disposal and patients' excreta. Thus, the safety risks of antitumor agents for patients with cancer who take medication at home after discharge cannot be ignored. Unsafe medication practices and errors-such as incorrect dosages or infusions, unclear instructions use of abbreviations and inappropriate prescriptions-are the leading causes of avoidable harm in health care around the world. Globally, the cost associated with medication errors has been estimated at US\$42 billion annually, not counting lost wages, productivity, or health care costs. This represents almost $1 \%$ of global expenditure on health (12). As the final gatekeeper of the safety of discharged patients with cancer, the pharmacists play an important role in the effectiveness and safety of subsequent treatment. Therefore, the purpose of this pilot study is to investigate the knowledge, attitude and practice (KAP) of Chinese hospital pharmacists towards the safety issues of antitumor agents for the patients with cancer discharged.

We present the following article in accordance with the SURGE reporting checklist (available at http://dx.doi. org/10.21037/tcr-20-2418).

\section{Methods}

\section{Study design}

This pilot study was conducted in 31 provinces (cities) in China. A method of convenience sampling was used for recruitment from March 15, 2019 to May 31, 2019. Interviewees were pharmacists of tertiary general hospital. They received regular inpatient pharmacist training and served more patients, and they had a good representation of the hospital pharmacist group. Fully consider the interviewees' different regions, different professional titles, different education levels, different working years and other conditions to ensure that there is no artificial bias.

\section{Questionnaire development}

After reviewing the related literature, a self-designed questionnaire was developed based on the KAP model and International Society of Oncology Pharmacy Practitioners (ISOPP) Standards of Practice. It was tested for practicality and ease of use on 10hospitalpharmacists working in a tertiary general hospital, feedback was collected, and adjustments then made. The questionnaire consisted of four sections:

\section{Demographic characteristics}

Socio-demographic and practice characteristics: gender, education level, working status and professional title.

Safety of patients with cancer who take medication at home after discharge related knowledge (hereinafter referred to as safety-related knowledge)

A safety-related knowledge questionnaire was used to assess the level of hospital pharmacists' perceived knowledge. Hospital pharmacists perceived knowledge score was assessed by ten questions evaluating: (I) what do you know about classification and pharmacology of antitumor drugs; (II) what do you know about dosage and application of antitumor drugs; (III) what do you know about route of administration of antitumor drugs; (IV) what do you know about solvent selection and infusion time of antitumor drugs; (V) how to cope with missed doses of antitumor drugs; (VI) what do you know about interactions between antitumor drugs and other drugs; (VII) what do you know about possible adverse reactions of antitumor drugs and countermeasures; (VIII) what do you know about storage and disposal of antitumor drugs; (IX) what do you know about the principles of safe handling, and dealing with spills and waste disposal; (X) how to search for information about antitumor drugs. Each question can be judged from Poor (0 point) to Excellent (10 points). The final score was calculated by summing the points obtained for all questions. 
The total possible score of this section was 100 points.

Safety of patients with cancer who take medication at home after discharge related attitudes (hereinafter referred to as safety-related attitudes)

The following five questionnaire items measured safetyrelated attitudes among hospital pharmacists: (I) Do you think there is a safety risk for patients with cancer who take medication at home after discharge (yes/neutral/no)? (II) Do you think it is important to give medication education to patients with cancer when discharged (yes/no)? (III) Do you think that medication education for patients with cancer at discharge can improve their compliance (improved/neutral/ unimproved)? (IV) Do you think that medication education for patients with cancer at discharge can improve patient safety (improved/neutral/unimproved)? (V) Would you like to provide medication education to patients with cancer when discharged (willing/neutral/unwilling)?

\section{Safety of patients with cancer who take medication} at home after discharge related practices (hereinafter referred to as safety-related practices)

The following four questionnaire items measured safetyrelated attitudes among hospital pharmacists: (I) Do you provide medication education to patients with cancer who take medication at home after discharge (yes/no)? (II) Whether the patients with cancer can contact you for medication consultation after discharge (yes/no). (III) Have you informed patients with cancer of possible adverse drug reactions and the ways to avoid and cope with them (yes/ no)? (IV) Have you informed patients with cancer which antitumor drug need special storage (yes/no)?

\section{Procedures}

The study was conducted in accordance with the Declaration of Helsinki (as revised in 2013). The study was approved by Peking Union Medical College Hospital Ethical Committee (NO.: S-K1309) and informed consent was taken from all the pharmacists. Then the KAP questionnaire was transformed into an online version using Wen Juan Xing (the earliest and the largest online questionnaire survey platform in China) and was able to access by participants on the website https://www.wjx. $\mathrm{cn} /$. The participation was voluntary and a link to the online questionnaire was given to hospital pharmacist via WeChat two-dimensional code. The second WeChat twodimensional code was sent to non-respondents 4 weeks after initial distribution and the survey was closed 6 weeks after the second invitation. Questionnaires filled in less than 1 minute are considered invalid. Participants could only submit one survey from their IP address. After the online survey respondents submit the questionnaire, the logical relationship between the questions and answers, and the integrity of the answers are reviewed, and invalid data or missing data are excluded. No monetary incentive or material award was provided for participation.

\section{Internal validity}

In the final questionnaire, the reliability of the safety-related knowledge section was evaluated by assessing the internal reliability and the Cronbach's $\alpha$ was 0.866 .

\section{Statistical analyses}

All data from completed questionnaires were collected automatically by the survey platform and downloaded into Microsoft Excel 2010 for statistical analysis with IBM SPSS 23.0 software (SPSS Inc., Chicago, IL, USA). Invalid data or missing data were excluded, and all data entries were double-checked to prevent errors. $T$-tests and ANOVA were employed to compare differences among continuous variables. There was a significant difference at $\mathrm{P}<0.05$, and a very significant difference at $\mathrm{P}<0.01$.

\section{Results}

\section{Characteristics of hospital pharmacists}

At the end of the data collection, 412 pharmacists accessed the online survey but only 360 completed it, 52 questionnaires were excluded because of missing data, resulting in a final sample of 360 for analysis (completion rate $87.4 \%$ ). Among the respondents, 94 male pharmacists $(26.1 \%)$ and 266 female pharmacists $(73.9 \%)$ were interviewed. About $62.2 \%$ of the participants had completed college education; and $35.3 \%$ had completed master degree; and $2.5 \%$ had completed $\mathrm{PhD}$ degree. Among them,49.2\% have served as hospital pharmacists for a decade or more, followed by pharmacists with 6-10 years of work experience $(30.0 \%)$. In addition, $42.0 \%$ of the participants were intermediate pharmacists (shown in Table 1).

\section{Safety-related knowledge}

Table 2 presented the average scores of safety-related 
Table 1 The demographic characteristics of hospital pharmacists $(\mathrm{n}=360)$

\begin{tabular}{lcc}
\hline Variables & $\mathrm{N}$ & $\%$ \\
\hline Gender & 94 & 26.1 \\
Male & 266 & 73.9 \\
Female & & \\
Education level & 18 & 5.0 \\
Junior college & 206 & 57.2 \\
Regular college & 127 & 35.3 \\
Master's degree & 9 & 2.5 \\
PhD degree & & \\
Working status & 75 & 20.8 \\
Below 5 years & 108 & 30.0 \\
6-10 years & 82 & 22.8 \\
11-20 years & 95 & 26.4 \\
Above 20 years & & \\
Professional title & 95 & 26.4 \\
Primary pharmacists & 70.4 \\
Intermediate pharmacist & & 42.0 \\
Associate chief pharmacist & & \\
Chief pharmacist & & \\
\hline & & \\
\hline
\end{tabular}

knowledge of hospital pharmacists based on gender, educational level, working status, and professional title. The total score of safety-related knowledge was 100 points; the mean score of safety-related knowledge was found to be 58.69 (SD: 19.04), which was equivalent to $58.7 \%$ of the total score. The average scores of ten questions were shown in Table 2. It was found that the lowest average score was in "Interactions between antitumor drugs and other drugs" and the highest average score was in "Search method of information about antitumor drugs". Statistically significant differences were found in gender, education level and working status (shown in Table 2).

\section{Safety-related attitudes}

The scores of hospital pharmacist's attitude towards the safety of patients with cancer who take medication at home after discharge were shown in Table 3. Most hospital pharmacists are neutral (57.8\%) about the safety risks of patients with cancer who take medication at home after discharge, while $32.2 \%$ of pharmacists clearly believe that safety risks exist and their safety-related knowledge scores were higher than those who believe that there are no safety risks $(\mathrm{P}<0.01)$.

The majority of pharmacists considered it important to give medication guidance to patients with cancer when they were discharged (95.3\%), and they were positive about the effect of medication guidance ("the compliance of patients", "the safety of medication ") (74.2\% and $76.7 \%$ respectively). At the same time, their total knowledge scores were significantly higher than those of negative pharmacists $(\mathrm{P}<0.01)$. It is encouraging that $85.3 \%$ of pharmacists were willing to provide medication guidance to patients with cancer discharged from hospitals if conditions permitted.

\section{Safety-related practices}

The survey results of the safety-related practices among hospital pharmacists are shown in Table 4. Only 15.6\% of the participants "never given medication guidance to discharged patients with cancer". The average scores of pharmacists who gave medication guidance to discharged patients with cancer were higher than those who did not. It is worth noting that the availability of hospital pharmacist for medication consultation after discharge is generally low (55.3\%). Most of the participants $(91.4 \%)$ have sometimes taken the initiative to promote safety-related knowledge (possible adverse effects and how to avoid and manage it) to their patients. The average scores in pharmacists who do have taken the initiative to promote safety-related knowledge to their patients were higher than those who had never $(8.6 \%)$. However, the safety-related knowledge scores of pharmacists who educated patients with cancer about the special storage conditions of antitumor drugs were not significantly different from those who did not.

The pilot study showed that hospital pharmacists' medication education for patients with cancer who take medication at home after discharge are mainly oral explanations $(62.1 \%)$ and written forms $(27.3 \%)$. Other safety-related practices for patients with cancer discharged include: WeChat drug education platform, electronic medicine guidance notes, public welfare talks, etc.

\section{Discussion}

The KAP model has been frequently applied in public health and health-related behavior changes. For example, to ensure safe and effective use of medicines, a KAP study in 


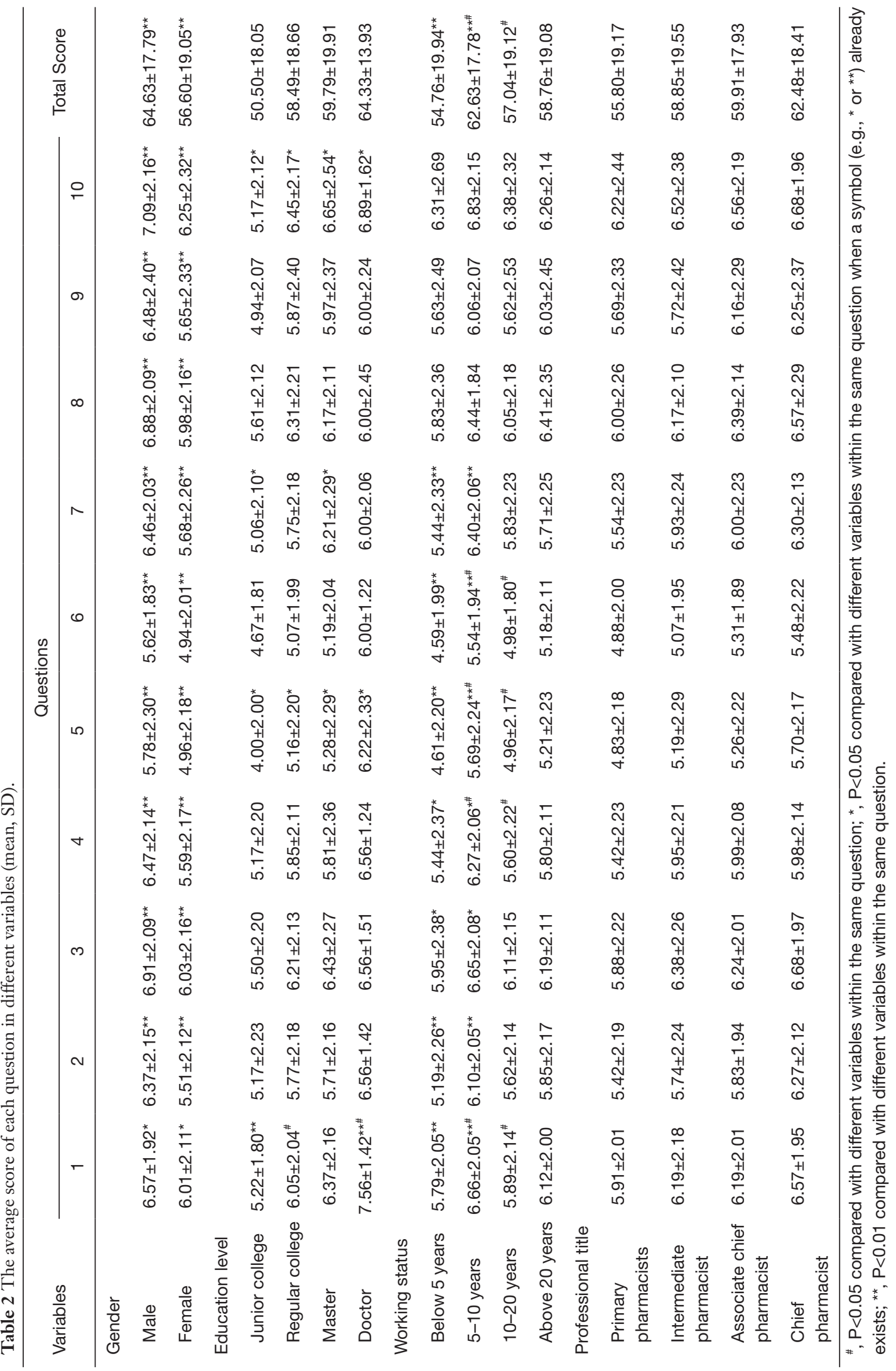


Table 3 The average score of each item in safety-related attitudes

\begin{tabular}{|c|c|c|c|c|c|}
\hline & Variables & $\mathrm{n}$ & $\%$ & $\begin{array}{l}\text { Total score of safety-related } \\
\text { knowledge (mean, SD) }\end{array}$ & $P$ \\
\hline \multirow[t]{3}{*}{1} & Yes & 116 & 32.2 & $67.41 \pm 16.27$ & $<0.01$ \\
\hline & Neutral & 208 & 57.8 & $57.96 \pm 17.20$ & \\
\hline & No & 36 & 10.0 & $34.89 \pm 16.14$ & \\
\hline \multirow[t]{3}{*}{3} & Improved & 267 & 74.2 & $61.86 \pm 18.38$ & 0.001 \\
\hline & Neutral & 88 & 24.4 & $51.09 \pm 17.30$ & \\
\hline & Unimproved & 5 & 1.4 & $23.60 \pm 8.62$ & \\
\hline \multirow[t]{3}{*}{5} & Willing & 307 & 85.3 & $60.83 \pm 18.48$ & 0.006 \\
\hline & Neutral & 49 & 13.6 & $48.18 \pm 16.76$ & \\
\hline & Unwilling & 4 & 1.1 & $23.50 \pm 11.90$ & \\
\hline
\end{tabular}

Table 4 The average score of each item in safety-related practices

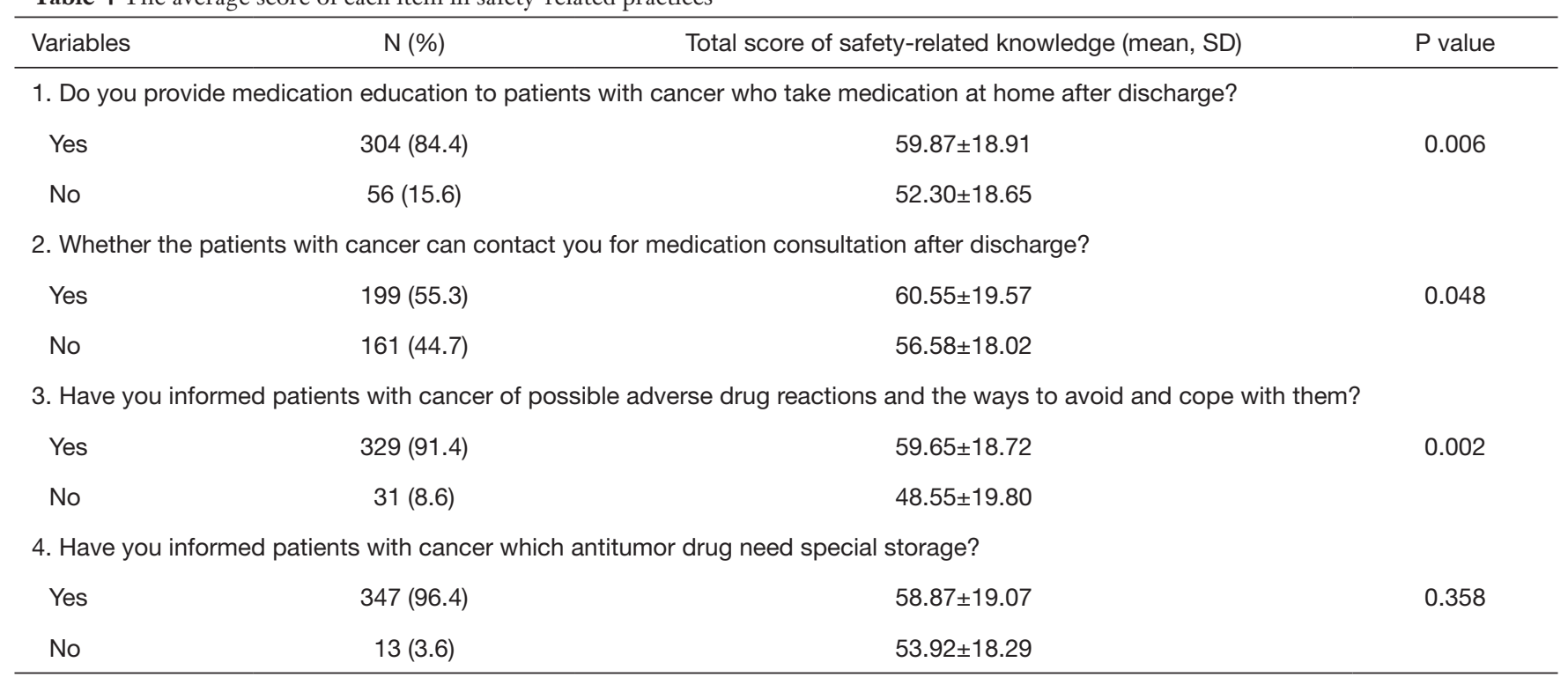

Malaysia provide some insights into the hospital pharmacists' KAP regarding medication therapy management (MTM) service. This model found that pharmacists had high level of knowledge and positive attitudes regarding MTM service and majority of them had willingness to become MTM service provider (13). Another KAP study showed that pharmacists have the overall required knowledge and attitude to play an important role in the patients' counseling towards dental care (14). Therefore, pharmacists may become important guide who can influence safety of patients discharged from hospital.

According to the basic principle of the KAP model, 
improving knowledge will change attitudes and behaviors to reduce the human and economic burden of diseases (15). This survey showed that the hospital pharmacists who participated had limited grasp of safetyrelated knowledge and the total score of safety-related knowledge among them was $58.7 \%$. A study by O'Leary et al. showed that roughly $80-95 \%$ of patients with breast tumors want to be informed and educated about their illness, treatment, and prognosis (16). Accuracy of patients' medication information is important to ensure patient safety (17). However, the insufficient knowledge of hospital pharmacists on antitumor drugs will affect the quality of drug safety information provided to patients with cancer, thus adversely affecting the safety of patients with cancer who take medication at home after discharge. Beside the transmission of patients' findings from physician to physician, the role of delivery of essential information to the patients for their safety is underestimated $(18,19)$. Fortunately, it was found that hospital pharmacists have a positive attitude towards the education of discharged patients with cancer and are willing to provide medication education to them when conditions permit.

This pilot study also showed that the safety risks of patients with cancer who take medication at home after discharge had not been regarded by hospital pharmacists (shown in Table 3, question 1). In a study by Sendlhofer et al., 360 risks were identified in hospital settings. From these, 176 risks were scored as strategic and clustered into "top risks", including medication errors, information errors, and lack of communication, among others (20). Therefore, the risks of patients with cancer who take medication at home after discharge needs to be taken seriously. In addition, the pilot study also found that hospital pharmacists barely support patients with their medicines after discharge (shown in Table4, question 2). This finding was in agreement with pervious study which reported that over a third of patients were unclear about what medicines they should be taking after discharge. This could be due to a lack of patient counselling, poor understanding of information or the patient not remembering information (21). Thus, attention should be paid to these factors to improve the pharmacists' practices regarding the safety issues of patients with cancer who take medication at home after discharge.

Previous studies by the authors have shown that there are indeed cytotoxic drugs in discharge medications for patients with cancer $(22,23)$, but there are few studies on how hospital pharmacists can do to effectively reduce the safety risks of these patients. To our knowledge, this pilot study is the first to explore the knowledge, attitude, practice of hospital pharmacists regard patients with cancer who take medication at home after discharge, and the demographic characteristics of respondents. By this pilot study, we found that hospital pharmacists have limited KAP on this issue and are not aware of the potential risks. In this case, it is necessary to strengthen the medication safety and environmental safety of patients with cancer discharged. This pilot study also verifies the feasibility of large-scale implementation of hospital pharmacist research on the safety issues of antitumor agents for the patients discharged and lays the foundation for possible further research. Research that focused on hospital pharmacists may play an important role in safety promotion of discharged patients with cancer, medication education, and development of a safe and high-quality oncology pharmacy service.

This pilot study has a few methodological limitations, including: (I) data obtained through this pilot study did not permit us to determine the causality; and (II) the limited sample (360 hospital pharmacists). Additionally, because this pilot study is a voluntary survey, the results may be skewed and do not represent the views of every hospital pharmacist in China.

\section{Conclusions}

This is the first known pilot study conducted to comprehensively evaluate the current status of KAP among the hospital pharmacists regarding to the safety issues of patients with cancer discharged in China. Highly attention should be paid to the knowledge of pharmacists, the awareness of the safety risks of patients with cancer who take medication at home after discharge, and the accessibility to consult with pharmacists after discharge in order to improve the safety of patients with cancer. There is still a long way to go for medication without harm for patients.

\section{Acknowledgments}

The authors wish to thank all the hospital pharmacists national wide for their participation and contribution.

Funding: None.

\section{Footnote}

Reporting Checklist: The authors have completed the 
SURGE reporting checklist. Available at http://dx. doi. org/10.21037/tcr-20-2418

Data Sharing Statement: available at http://dx.doi. org/10.21037/tcr-20-2418

Conflicts of Interest: All authors have completed the ICMJE uniform disclosure form (available at http://dx.doi. org/10.21037/tcr-20-2418). The authors have no conflicts of interest to declare.

Ethical Statement: The authors are accountable for all aspects of the work in ensuring that questions related to the accuracy or integrity of any part of the work are appropriately investigated and resolved. The study was conducted in accordance with the Declaration of Helsinki (as revised in 2013). The study was approved by Peking Union Medical College Hospital Ethical Committee (NO.: S-K1309) and informed consent was taken from all the pharmacists.

Open Access Statement: This is an Open Access article distributed in accordance with the Creative Commons Attribution-NonCommercial-NoDerivs 4.0 International License (CC BY-NC-ND 4.0), which permits the noncommercial replication and distribution of the article with the strict proviso that no changes or edits are made and the original work is properly cited (including links to both the formal publication through the relevant DOI and the license). See: https://creativecommons.org/licenses/by-nc-nd/4.0/.

\section{References}

1. Cao MM, Chen WQ. Epidemiology of cancer in China and the current status of prevention and control. Chin J Clin Oncol 2019;46:145-9.

2. GLOBOCAN. Population Fact Sheets. GLOBOCAN, 2019. [2020-01-03]. Available online: https://gco.iarc. fr/today/data/factsheets/populations/160-china-factsheets.pdf

3. Mitra AK, Agrahari V, Mandal A, et al. Novel delivery approaches for cancer therapeutics. J Control Release 2015;219:248-68.

4. Liu CG, Han YH, Kankala RK, Wang SB, Chen AZ. Subcellular Performance of Nanoparticles in Cancer Therapy. Int J Nanomedicine 2020;15:675-704.

5. Mohammadinejad R, Dehshahri A, Sagar Madamsetty V, et al. In vivo gene delivery mediated by non-viral vectors for cancer therapy. J Control Release 2020;325:249-275.

6. Murciano-Goroff YR, Warner AB, Wolchok JD. The future of cancer immunotherapy: microenvironmenttargeting combinations. Cell Res 2020;30:507-19.

7. WHO. Cancer. Available online: https://www.who.int/ health-topics/cancer\#tab=tab_1

8. Gao F, Teng F, Liang H, et al. Post discharge care guidance needs of cancer patients. Chinese Journal of Clinical Oncology and Rehabilitation 2019;26:498-501.

9. International Society of Oncology Pharmacy Practicioners Standards Committee. ISOPP standards of practice. Safe handling of cytotoxics. J Oncol Pharm Pract 2007;13 Suppl:1-81.

10. Keers RN, Williams SD, Cooke J, Ashcroft DM. Causes of medication administration errors in hospitals: a systematic review of quantitative and qualitative evidence. Drug Saf. 2013;36:1045-67.

11. Institute for Safe Medication Practices. ISMP's List of High-Alert Medications.

12. WHO. 10 facts on patient safety. Available online: https:// www.who.int/features/factfiles/patient_safety/en/

13. Al-Tameemi NK, Sarriff A. Knowledge, attitude and practice of pharmacists on medication therapy management: a survey in Hospital Pulau Pinang, Penang, Malaysia. J Pharm Health Care Sci 2019;5:1.

14. Hajj A, Hallit S, Azzo C, et al. Assessment of knowledge, attitude and practice among community pharmacists towards dental care: A national cross sectional survey. Saudi Pharm J 2019;27:475-83.

15. Xu X., Chen C, Abdullah AS, et al. Smoking related attitudes, motives, and behaviors of male secondary school students in an urban setting of China. Springerplus 2016;5:2021.

16. O'Leary KA, Estabrooks CA, Olson K,et al. Information acquisition for women facing surgical treatment for breast cancer: influencing factors and selected outcomes. Patient Educ Couns 2007;69:5-19.

17. Tong EY, Roman CP, Mitra B, et al. Reducing medication errors in hospital discharge summaries: a randomized controlled trial. Med J Aust 2017;206:36-9.

18. Lin R, Tofler G, Spinaze M, et al. Patient-directed discharge letter (PADDLE)-a simple and brief intervention to improve patient knowledge and understanding at time of hospital discharge. Hear Lung Circ 2012;21:S312.

19. Yemm R, Bhattacharya D, Wright $D$, et al. What constitutes a high-quality discharge summary? A comparison between the views of secondary and primary care doctors. Int J Med Educ 2014;5:125-31. 
20. Sendlhofer G, Brunner G, Tax C, et al. Systematic implementation of clinical risk management in a large university hospital: the impact of risk managers. Wien Klin Wochenschr 2015;127:1-11.

21. Wright S, Morecroft CW, Mullen R, et al. UK hospital patient discharge: the patient perspective. Eur J Hosp Pharm 2017;24:338-42.

Cite this article as: $\mathrm{Xu} \mathrm{X}$, Yang Y, Fan Q, Zhu Z. The knowledge, attitude and practice of hospital pharmacists on the safety issues of antitumor agents for the patients discharged in China. Transl Cancer Res 2021;10(1):201-209. doi: 10.21037/tcr20-2418
22. Fan QQ, Chang Q, Zhu Z, et al. Application and risk analysis on the anticancer drugs prescribed for discharged cancer patients. Chinese Journal of Drug Application and Monitoring 2019;5:297-301.

23. Liu F, Zhang XL, Zhu Z, et al. Strengthen the Strategy to Prevent Errors in the Use of High-alert Drugs. Adverse Drug Reactions Journal 2018;20:321-3. 